\title{
Metric Space Structures for Computational Anatomy
}

\author{
Jianqiao Feng ${ }^{1,2}$, Xiaoying Tang ${ }^{1,2}$, Minh Tang ${ }^{1,3}$, \\ Carey Priebe ${ }^{1,3}$, and Michael Miller ${ }^{1,4}$ \\ 1 Center of Imaging Science, The Johns Hopkins University, United States \\ 2 Department of Electrical and Computer Engineering, \\ The Johns Hopkins University, United States \\ 3 Department of Applied Mathematics and Statistics, \\ The Johns Hopkins University, United States \\ 4 Department of Biomedical Engineering, \\ The Johns Hopkins University, United States
}

\begin{abstract}
This paper describes a method based on metric structures for anatomical analysis on a large set of brain MR images. A geodesic distance between each pair was measured using large deformation diffeomorphic metric mapping (LDDMM). Manifold learning approaches were applied to seek a low-dimensional embedding in the highdimensional shape space, in which inference between healthy control and disease groups can be done using standard classification algorithms. In particular, the proposed method was evaluated on ADNI, a dataset for Alzheimer's disease study. Our work demonstrates that the highdimensional anatomical shape space of the amygdala and hippocampi can be approximated by a relatively low dimension manifold.
\end{abstract}

Keywords: structural MR image, computational anatomy, Alzheimer's disease, manifold learning, shape analysis.

\section{Introduction}

In the past decade, computational anatomy (CA) [1] has emerged as a discipline to study the neuroanatomical variability via morphometric mapping algorithms. Quantitative analysis of anatomy has thus become possible. This paper studies the problem of embedding healthy control (HC) and Alzheimer's disease (AD) subjects into an anatomical shape space by computing a dissimilarity representation between subjects. Manifold learning techniques are applied on the dissimilarity representation to obtain embeddings for different subjects followed by classification in the embedded space. A widely-used framework in CA, large deformation diffeomorphic metric mapping (LDDMM) 2] was used for dissimilarity measurement. The proposed method was evaluated on a dataset of $385 \mathrm{MR}$ images obtained from the Alzheimer's Disease Neuroimaging Initiative (ADNI) [3]. Instead of studying the whole brain, here we consider only two anatomical structures, hippocampus and amygdala, which have been reported to be affected morphologically by AD.

G. Wu et al. (Eds.): MLMI 2013, LNCS 8184, pp. 123-130, 2013.

(C) Springer International Publishing Switzerland 2013 
There have been several related methods proposed in the literature to apply manifold learning methods to dissimilarities measured on structural images. Most of them quantify inter-subject dissimilarity based on volume image data. For example, a similarity measurement called bending energy is employed in [4]. In [5, 6], random forest has been used to measure dissimilarity on some statistical region-based features extracted from volume images. In [7], dissimilarity was calculated via a small deformation to approximate a large deformation for computational efficiency. Similar approximations can be found in [8, 9, 4]. However, the explosive growth of computing power along with parallel computing resources have made the problem of computation less severe. Thus a full large deformation diffeomorphic metric is adopted in this paper. In this study, we couple the volume imagery to surfaces, and track the variations of shape using surface models. Studying surfaces allows us to capture the variation of neurodevelopment very efficiently [8].

Low-dimensional embedding can be calculated from the dissimilarity information via standard manifold learning approaches, such as multidimensional scaling (MDS) used in [5, 6, 10], Isomap used in [4, 8, 9], Laplacian Eigenmaps (LE) used in [7].

After the embedding in low-dimensional space is calculated, statistical inference is often carried out. One choice is classification between different cohorts. Classification accuracy then becomes an important criteria for evaluating the dimension of the embedding. We note that we only consider the two-class classification problem, i.e., HC and AD. In this paper, a number of standard manifold learning and classification algorithms were employed. Section 3 presents our experiments and result. Compared with related works mentioned above, our investigations are more extensive, i.e., we consider a larger dataset. Our methodology achieves comparable classification accuracy.

\section{Methods}

In this section, the framework of our method is described step by step. The flowchart is presented in Figure 1 .

\subsection{Data Acquisition}

The dataset used in our experiment contains $385 \mathrm{~T} 1$ weighted MR images obtained from ADNI [3]. Although there are more than 800 subjects with 4000 scans in ADNI database, we considered the healthy control and disease groups, with the baseline, i.e. the first scan, for each subject. Some scans (84 out of 840) were excluded if they suffered severe degradation due to motion artifacts or significant clinical abnormalities (e.g., hemispheric infarction). A dataset of 756 subjects was formed after this unbiased selection, including 210 subjects of $\mathrm{HC}$, 175 subjects of $\mathrm{AD}$, and 371 subjects of Mild Cognitive Impairment (MCI). We only considered the $\mathrm{HC}$ and $\mathrm{AD}$ here. Table 1 presents detailed information of this dataset. 

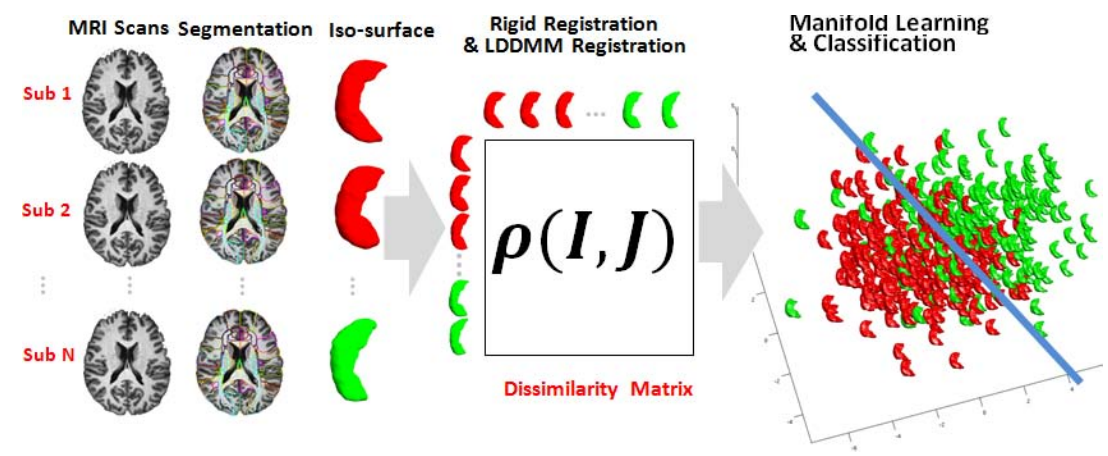

Fig. 1. The flowchart of the framework: data acquisition, segmentation, surface extraction, rigid registration, LDDMM-surface mapping, manifold learning, and classification

Table 1. Demographic characteristics of the dataset used in this paper

\begin{tabular}{c|c|c}
\hline Group & HC & AD \\
\hline number of subjects & 210 & 175 \\
\hline number of male subjects & 109 & 94 \\
\hline Age (year) & $76.25 \pm 5.01$ & $75.28 \pm 7.49$ \\
\hline
\end{tabular}

\subsection{Preprocessing: Segmentation and Iso-surface Extraction}

We followed a similar procedure as used in [11], in which template surfaces are used to initialize the topology of the subcortical structures upon which the inference will be performed, and then target surfaces are inserted into the segmentations from Freesurfer [12, 13] via LDDMM mapping. These transformed template surfaces towards different target surfaces are accurate since the LDDMM mappings used are smooth, and mediate the noise which may be sometimes inherent in segmentations. These surfaces then become the manifolds that our inference proceeds based on.

\subsection{Diffeomorphic Metric Mapping}

To measure dissimilarity between two subjects, diffeomorphic metric mapping is calculated via LDDMM surface mapping [14, 15]. The diffeomorphisms $\phi_{t}$ are constructed as a flow of ordinary differential equation:

$$
\dot{\phi}_{t}=v_{t}\left(\phi_{t}\right), \phi_{0}=I d, t \in[0,1] \text {, }
$$

where $v_{t}$ is the velocity vector field which determines the corresponding flow $\phi_{t}$. The boundary value of this ODE is the identity map denoted as $I d$.

The LDDMM surface mapping algorithm seeks the optimal velocity field to minimize a loss function combining smoothness and goodness of fit of the mapping $\phi$. Given a pair of surfaces $I$ and $J$, a dissimilarity $\rho(I, J)$ between them is calculated by integrating the norm of velocity vector field associated with the 
geodesic $\phi_{t}$ over time, where $\sigma$ is the parameter for trade-off between smoothness and goodness of fit.

$$
\rho(I, J)=\int_{0}^{1} \| v_{t}^{*} \mid d t, \text { where } v_{t}^{*}=\arg \min _{v \in V} \int_{0}^{1}\left\|v_{t}\right\|_{v} d t+\frac{1}{\sigma^{2}}\left\|I \cdot \phi_{1}^{-1}-J\right\|_{L^{2}}
$$

The second term, goodness of fit, implies this is not an exact matching problem, because the exact matching problem does not have a well defined solution. Thus $\rho(I, J)$ is not a precise metric for it is not symmetric, and this effect has been studied in [16]. Here we took the "averaging" strategy, i.e. $\rho^{\prime}(I, J)=\rho^{\prime}(J, I) \triangleq \frac{(\rho(I, J)+\rho(J, I))}{2}$, to make it symmetric. In addition, prior to LDDMM a rigid registration was carried out to remove the variation caused by different poses in image acquisition stage.

\subsection{Manifold Learning and Classification}

Manifold learning is a popular approach of non-linear dimension-reduction. It attempts to find a low-dimensional embedding (i.e., the manifold) in the highdimensional space. The hypothesis is that the data points are samples from a low-dimensional manifold. There are a number of algorithms in the manifold learning family. Here we consider three of them, i.e., classical MDS, Isomap [17], and Laplacian Eigenmaps (LE) [18].

Four widely used classification algorithms, K-Nearest Neighbor (KNN) and Fisher's linear discriminant analysis (LDA), Support Vector Machine (SVM), and Random Forest (RF) were employed to discriminate $\mathrm{HC}$ and $\mathrm{AD}$ cohorts.

For SVM, LIBSVM was used [19]. Beside the original linear SVM, another nonlinear kernel function, Radial Basis, was tried. Random forest [20] is an extension of traditional decision tree, which makes an overall prediction based on decisions of all individual trees.

\section{$3 \quad$ Experiments and Results}

We tested the proposed method on two anatomical structures, the hippocampus and amygdala. For each subject, four surfaces including both left and right sides are studied. On any of these surfaces, an inter-subject dissimilarity was calculated via LDDMM following a rigid registration as described in 2.3 .

After surface mapping finished, one of three manifold learning methods, MDS, Isomap, and Laplacian Eigenmaps, was employed to calculate embedding, on which several classifiers were trained and tested. A 6-fold cross-validation was carried out to evaluate the performance on 385 subjects (210 HC and $175 \mathrm{AD}$ ). The performance was measured using misclassification rate.

In order to understand whether the dimension of representation for the anatomical shape space is large or small, misclassification rate was calculated by varying the dimensions of the embedding. In particular, we considered the first $d$ dimensions corresponding to the largest $d$ eigen-values for $d=1, \ldots 90$. 


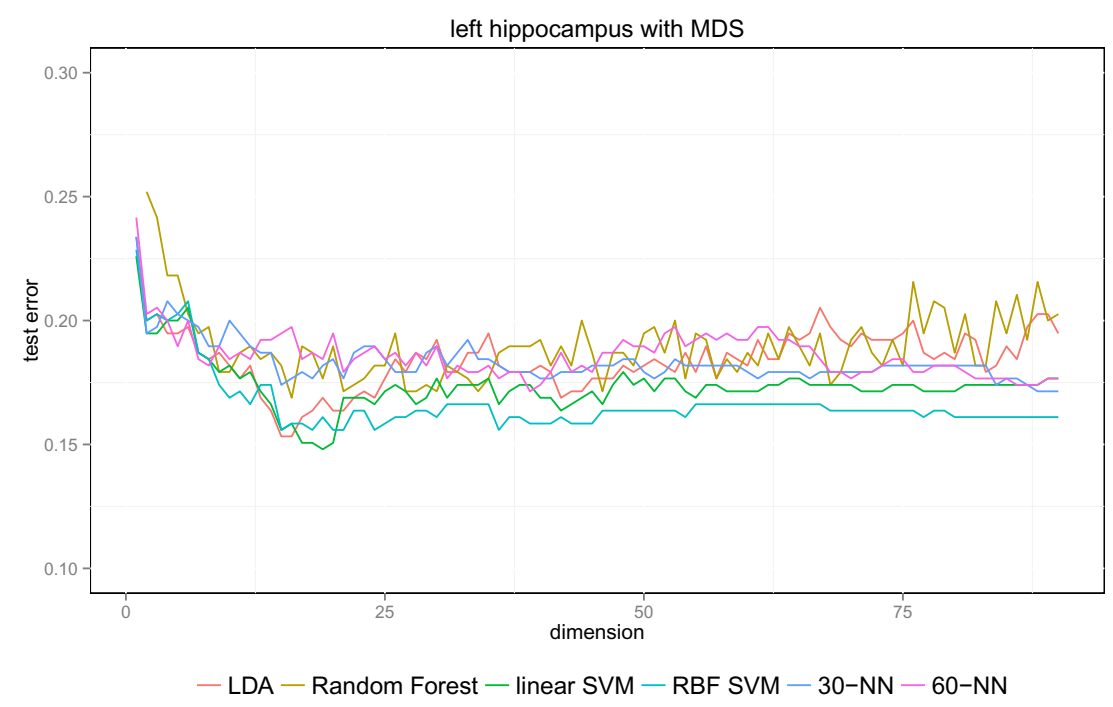

Fig. 2. Misclassification rate as a measure of the embedding dimensions from the left hippocampus via MDS

As a result, a curve of misclassification rates over different dimensions was obtained for each classification algorithm. Figure 2 presents misclassification rates, using MDS followed by a certain classification algorithm, as a function of the number of embedding dimensions calculated from the left hippocampus. From the misclassification curves, one can observe that the misclassification rate first decreases rapidly then increases slowly, as the embedding dimensions increases. Thus, a low dimension embedding is sufficient to achieve a low misclassification rate. However, increasing the dimensionality can lead to noises and over-fitting. The dimensions with lowest misclassification rate under our setup, is 15 to 20 for most classification algorithms approximately. This suggests that anatomical shape lies in a space of relatively low dimensionality. Other combinations of \{MDS, Isomap, Laplacian Eigenmaps $\}$ and \{left/right hippocampus, left/right amygdala $\}$ give similar results and are not reported here due to space constraints.

Table 2 is a full comparison of minimum misclassification rates and the corresponding number of dimensions from different embedding algorithms and classification algorithms (on left/right hippocampus). In that table, $S V M_{L}$ indicates SVM using linear kernel (original version). $S V M_{R}$ represents the radial basis kernel. RF represents random forest. The number of dimension corresponding to minimum misclassification rates, are highlighted in each column. The standard error for each of entry of Table 2 is approximately one percent. The minimum misclassification rate is around $14 \%$ which is achieved via Laplacian Eigenmaps on the left hippocampus. Lower misclassification rates are achieved on the left hippocampus than on right hippocampus regardless of which manifold learning algorithm 
is used. Similar phenomena can be observed for the amygdala. Manifold learning algorithms, MDS, Isomap, and Laplacian Eigenmaps give comparable results.

Table 3 is a summary comparison of performance reported in related works. Note that it only lists the methods strongly similar to our methodology, i.e., computation of dissimilarity followed by manifold learning and classification. It is difficult to compare the classification accuracy directly with other published methods, given that the difference may be caused by the different datasets used (different database or different subset of ADNI). Our embedding achieves classification accuracy comparable with related works.

Table 2. Smallest Misclassification rate $\mathrm{L}^{*}$ and the corresponding dimension $\mathrm{d}^{*}$

\begin{tabular}{|c|c|c|c|c|c|c|c|c|c|c|c|c|}
\hline \multirow{3}{*}{ Classifier } & \multicolumn{6}{|c|}{ Left Hippocampus } & \multicolumn{6}{|c|}{ Right Hippocampus } \\
\hline & \multicolumn{2}{|c|}{ Isomap } & \multicolumn{2}{|c|}{ MDS } & \multicolumn{2}{|l|}{$\mathrm{LE}$} & \multicolumn{2}{|c|}{ Isomap } & \multicolumn{2}{|c|}{ MDS } & \multicolumn{2}{|l|}{ LE } \\
\hline & $\mathrm{L}^{*}$ & $\mathrm{~d}^{*}$ & $\mathrm{~L}^{*}$ & $d^{*}$ & $\mathrm{~L}^{*}$ & a & $\mathrm{L}^{*}$ & $d^{*}$ & $\mathrm{~L}^{*}$ & $d^{*}$ & $\mathrm{~L}^{*}$ & $d^{*}$ \\
\hline LDA & $18.7 \%$ & 9 & $15.3 \%$ & 15 & $14.0 \%$ & 26 & $20.2 \%$ & 7 & $19.5 \%$ & 25 & $21.3 \%$ & 20 \\
\hline $\mathrm{RF}$ & $19.2 \%$ & 65 & $16.9 \%$ & 16 & $17.9 \%$ & 27 & $21.3 \%$ & 13 & $21.3 \%$ & 33 & $22.6 \%$ & 6 \\
\hline$S V M_{L}$ & $18.2 \%$ & 53 & $14.8 \%$ & 19 & $14.8 \%$ & 42 & $19.2 \%$ & 17 & $19.2 \%$ & 27 & $22.1 \%$ & 3 \\
\hline$\overline{S V M_{R}}$ & $17.4 \%$ & 53 & $15.6 \%$ & 15 & $15.6 \%$ & 25 & $18.9 \%$ & 38 & $20.8 \%$ & 16 & $22.3 \%$ & 3 \\
\hline $30-\mathrm{NN}$ & $19.2 \%$ & 24 & $17.1 \%$ & 88 & $18.9 \%$ & 13 & $21.6 \%$ & 20 & $21.3 \%$ & 55 & $22.3 \%$ & 3 \\
\hline $60-\mathrm{NN}$ & $17.7 \%$ & 43 & $17.1 \%$ & 39 & $18.4 \%$ & 7 & $19.5 \%$ & 26 & $22.1 \%$ & 18 & $21.0 \%$ & $\mathbf{9}$ \\
\hline
\end{tabular}

Table 3. Summary of representative methods in the literature ${ }^{1}$

\begin{tabular}{c|c|c|c|c|c|c}
\hline Method & {$[4]$} & {$[5]$} & {$[6]$} & {$[7]$} & {$[10]$} & Our method \\
\hline \{\#subjects of HC\}/\{\#subject of AD & $25 / 25$ & $69 / 71$ & $37 / 35$ & $116 / 103$ & $57 / 44$ & $210 / 175$ \\
\hline Accuracy & $76-84 \%$ & $87 \%$ & $83 \%$ & $86 \%$ & $77 \%$ & $85 \%$ \\
\hline
\end{tabular}

\subsection{Comparison to Template Based Classifiers}

In the framework of LDDMM, we calculated a geodesic from the template coordinate space to the target coordinates. The method proposed in this paper is template-free, since there does not exist a fixed template. For template-based methods, given a fixed template, the anatomical variability within a group of subjects is encoded via the geodesic. In [21], computational tools are provided for comparing these geodesic transformations and derived a fundamental "conservation of momentum" property of these geodesics: the initial momentum encodes the geodesic connecting the template to the subject. Anatomical differences among different target groups can, therefore, be studied by analyzing the initial momentum associated with different subjects. In [22], the initial momentum space is demonstrated to be linear, and thus linear statistical analysis such as the principal component analysis (PCA) can be applied to that space. Another paper [23] successfully utilized the initial momentum space associated with volume to discriminate disease groups. In our experiment, we combined

\footnotetext{
${ }^{1}$ The accuracy listed here is only that based on MR images (one scan for each subject).
} 
PCA and LDA to differentiate HC and AD. According to the cross-validation results, we observed, in terms of misclassification rate, $15 \%, 19 \%, 20 \%$, and $21 \%$ respectively from the PCA+LDA procedure applied on the initial momentum space of left hippocampus, right hippocampus, left amygdala, and right amygdala. This implies the performance of our template-free method proposed in this paper is comparable to the template-based classification method.

\section{Conclusion}

In this paper, we present a framework of embedding anatomical shape information into a low-dimensional space and discriminating subjects with AD from healthy controls using various classification algorithms. The LDDMM algorithm was used for measuring dissimilarity between every pair of anatomical shapes. This is in some sense a special way to extract biomarkers from MR image data. This framework achieves the comparable performance with similar application of manifold learning in discriminating subjects with cognitive dementia from healthy controls. A potential application or extension of our work is to combine the shape information extracted using this method and other imaging features extracted from different imaging modalities with their fusion improving classification accuracy [5 7$]$.

Our result suggests that a suitable representation of anatomical shape space is inherently of low dimension. Another conclusion can be drawn from the result is that the key step in this manifold learning framework is the dissimilarity measurement because as shown in Section 3 similar results can be achieved when different manifold learning or classification algorithms were used.

\section{References}

1. Grenander, U., Miller, M.: Computational anatomy: An emerging discipline. Quarterly of Applied Mathematics 56(4), 617-694 (1998)

2. Beg, M., Miller, M., Trouvé, A., Younes, L.: Computing large deformation metric mappings via geodesic flows of diffeomorphisms. International Journal of Computer Vision 61(2), 139-157 (2005)

3. Mueller, S., Weiner, M., Thal, L., Petersen, R., Jack, C., Jagust, W., Trojanowski, J., Toga, A., Beckett, L.: The Alzheimers disease neuroimaging initiative. Neuroimaging Clinics of North America 15(4), 869 (2005)

4. Park, H.: Isomap induced manifold embedding and its application to Alzheimer's disease and mild cognitive impairment. Neuroscience Letters (2012)

5. Gray, K.R., Aljabar, P., Heckemann, R.A., Hammers, A., Rueckert, D.: Random forest-based manifold learning for classification of imaging data in dementia. In: Suzuki, K., Wang, F., Shen, D., Yan, P. (eds.) MLMI 2011. LNCS, vol. 7009, pp. 159-166. Springer, Heidelberg (2011)

6. Gray, K., Aljabar, P., Heckemann, R., Hammers, A., Rueckert, D.: Random forestbased similarity measures for multi-modal classification of Alzheimer's disease. NeuroImage (2012)

7. Wolz, R., Aljabar, P., Hajnal, J., Lötjönen, J., Rueckert, D.: Nonlinear dimensionality reduction combining $\mathrm{mr}$ imaging with non-imaging information. Medical Image Analysis (2011) 
8. Yang, X., Goh, A., Qiu, A.: Approximations of the diffeomorphic metric and their applications in shape learning. In: Székely, G., Hahn, H.K. (eds.) IPMI 2011. LNCS, vol. 6801, pp. 257-270. Springer, Heidelberg (2011)

9. Gerber, S., Tasdizen, T., Fletcher, P., Joshi, S., Whitaker, R.: Manifold modeling for brain population analysis. Medical Image Analysis 14(5), 643 (2010)

10. Miller, M., Priebe, C., Qiu, A., Fischl, B., Kolasny, A., Brown, T., Park, Y., Ratnanather, J., Busa, E., Jovicich, J., et al.: Collaborative computational anatomy: an mri morphometry study of the human brain via diffeomorphic metric mapping. Human Brain Mapping 30(7), 2132-2141 (2008)

11. Qiu, A., Miller, M.I., et al.: Multi-structure network shape analysis via normal surface momentum maps. NeuroImage 42(4), 1430 (2008)

12. Fischl, B., Salat, D., Busa, E., Albert, M., Dieterich, M., Haselgrove, C., van der Kouwe, A., Killiany, R., Kennedy, D., Klaveness, S., et al.: Whole brain segmentation: automated labeling of neuroanatomical structures in the human brain. Neuron 33(3), 341-355 (2002)

13. Khan, A.R., Wang, L., Beg, M.F.: Freesurfer-initiated fully-automated subcortical brain segmentation in mri using large deformation diffeomorphic metric mapping. NeuroImage 41(3), 735 (2008)

14. Vaillant, M., Qiu, A., Glaunès, J., Miller, M.: Diffeomorphic metric surface mapping in subregion of the superior temporal gyrus. NeuroImage 34(3), 1149-1159 (2007)

15. Vaillant, M., Glaunès, J.: Surface matching via currents. In: Christensen, G.E., Sonka, M. (eds.) IPMI 2005. LNCS, vol. 3565, pp. 381-392. Springer, Heidelberg (2005)

16. Trosset, M.W., Priebe, C.E., Park, Y., Miller, M.I.: Semisupervised learning from dissimilarity data. Computational Statistics \& Data Analysis 52(10), 4643-4657 (2008)

17. Tenenbaum, J., De Silva, V., Langford, J.: A global geometric framework for nonlinear dimensionality reduction. Science 290(5500), 2319-2323 (2000)

18. Belkin, M., Niyogi, P.: Laplacian eigenmaps and spectral techniques for embedding and clustering. In: Advances in Neural Information Processing Systems, vol. 14, pp. 585-591 (2001)

19. Chang, C.C., Lin, C.J.: LIBSVM: A library for support vector machines. ACM Transactions on Intelligent Systems and Technology 2, 27:1-27:27 (2011), Software available at http://www.csie.ntu.edu.tw/ cjlin/libsvm

20. Liaw, A., Wiener, M.: Classification and regression by randomforest. R News 2(3), 18-22 (2002)

21. Miller, M., Trouvé, A., Younes, L.: Geodesic shooting for computational anatomy. Journal of Mathematical Imaging and Vision 24(2), 209-228 (2006)

22. Vaillant, M., Miller, M.I., Younes, L., Trouvé, A., et al.: Statistics on diffeomorphisms via tangent space representations. NeuroImage 23(1), 161 (2004)

23. Wang, L., Beg, F., Ratnanather, T., Ceritoglu, C., Younes, L., Morris, J.C., Csernansky, J.G., Miller, M.I.: Large deformation diffeomorphism and momentum based hippocampal shape discrimination in dementia of the Alzheimer type. IEEE Transactions on Medical Imaging 26(4), 462-470 (2007) 